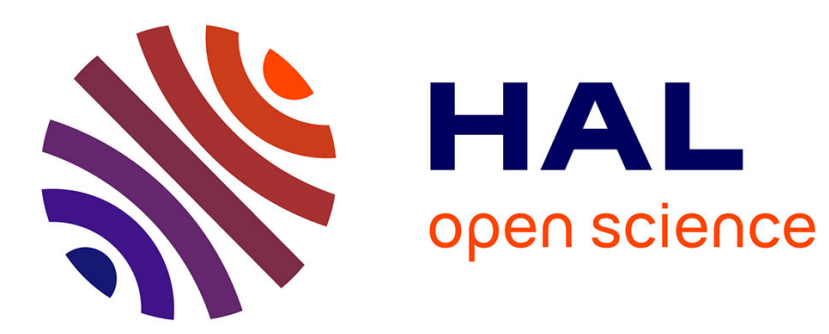

\title{
A Finite-Volume Approach to 1D Nonlinear Elastic Waves: Application to Slow Dynamics
}

H Berjamin, Bruno Lombard, Guillaume Chiavassa, Nicolas Favrie

\section{To cite this version:}

H Berjamin, Bruno Lombard, Guillaume Chiavassa, Nicolas Favrie. A Finite-Volume Approach to 1D Nonlinear Elastic Waves: Application to Slow Dynamics. Acta Acustica united with Acustica, 2018, 10.3813/Aaa.919197 . hal-02111888

\section{HAL Id: hal-02111888 https://hal-amu.archives-ouvertes.fr/hal-02111888}

Submitted on 26 Apr 2019

HAL is a multi-disciplinary open access archive for the deposit and dissemination of scientific research documents, whether they are published or not. The documents may come from teaching and research institutions in France or abroad, or from public or private research centers.
L'archive ouverte pluridisciplinaire HAL, est destinée au dépôt et à la diffusion de documents scientifiques de niveau recherche, publiés ou non, émanant des établissements d'enseignement et de recherche français ou étrangers, des laboratoires publics ou privés. 


\title{
A Finite-Volume Approach to 1D Nonlinear Elastic Waves: Application to Slow Dynamics
}

\author{
H. Berjamin ${ }^{1)}$, B. Lombard ${ }^{1)}$, G. Chiavassa ${ }^{2)}$, N. Favrie ${ }^{3)}$ \\ 1) Aix-Marseille Univ, CNRS, Centrale Marseille, LMA, Marseille, France. lombard@1ma.cnrs-mrs.fr \\ 2) Centrale Marseille, CNRS, Aix-Marseille Univ, M2P2 UMR 7340, 13451 Marseille Cedex 20, France \\ 3) Aix-Marseille Univ, UMR CNRS 7343, IUSTI, Polytech Marseille, 13453 Marseille Cedex 13, France
}

\begin{abstract}
Summary
A numerical method for longitudinal wave propagation in nonlinear elastic solids is presented. Here, we consider polynomial stress-strain relationships, which are widely used in nondestructive evaluation. The large-strain and infinitesimal-strain constitutive laws deduced from Murnaghan's law are detailed, and polynomial expressions are obtained. The Lagrangian equations of motion yield a hyperbolic system of conservation laws. The latter is solved numerically using a finite-volume method with flux limiters based on Roe linearization. The method is tested on the Riemann problem, which yields nonsmooth solutions. The method is then applied to a continuum model with one scalar internal variable, accounting for the softening of the material (slow dynamics).
\end{abstract}

PACS no. 43.25.Dc, 02.70.Bf

\section{Introduction}

Rocks and concrete are known to behave nonlinearly when vibrating longitudinally, even at very low amplitudes $[1,2]$. Firstly, dynamic acoustoelastic testing (DAET) $[3,4]$ reveals that the speed of sound measured locally decreases with time, and recovers its initial value after the excitation is stopped. This softening occurs over a time scale larger than the period of the dynamic loading, which highlights the phenomenon of slow dynamics. Secondly, the evolution of this speed with respect to the strain presents an hysteretical behavior. Lastly, all these phenomena are enhanced when the forcing amplitude is increased. Such observations are not compatible with linear elastodynamics, where the speed of sound is a constant.

Polynomial nonlinear stress-strain relationships are widely used in nondestructive testing [5, 6]. However, nonlinear elasticity is not sufficient to represent the softening and the hysteresis revealed by DAET. Several models can be found in the literature to reproduce these phenomena (see e.g. [7, 8]). The soft-ratchet model by Vakhnenko et al. $[9,10]$ consists in introducing a scalar variable $g$ to describe the softening of the material. A similar model with refinements was proposed by Lyakhovsky and coauthors in a series of papers $[11,12]$. Recently, a 3D model of continuum has been developed by the authors [13] in the framework of continuum thermodynamics with internal variables of state $[14,15]$. This model, which generalizes the soft-ratchet model to 3D geometries and fixes thermodynamical issues, will be used along the present paper.
Regardless the fact that slow dynamics is taken into account or not, the equations of the model appear as a nonlinear hyperbolic system of conservation laws with relaxation, the solutions of which may be discontinuous (shock waves). The numerical computation of nonsmooth solutions requires a particular care. Here, dedicated finitevolume methods $[16,17,18]$ are used to compute such solutions in a non-oscillatory manner. A 1D scheme based on the Roe linearization and flux limiters is adapted to the present system of equations. Due to the separation of timescales between the wave propagation and the softening of the material, the coupling between nonlinear elastodynamics and the slow dynamics is straightforward.

The article is organized as follows. For pedagogical purposes, the case of nonlinear elastic solids without slow dynamics is first considered (sections 2 and 3). Section 2 derives the equations of nonlinear elastodynamics, and the case of Murnaghan hyperelastic material [19] is tackled. The use of this law is discussed, in particular the way it relates to polynomial stress-strain relationships [20, 21]. The numerical method is presented in section 3, including the construction of a Roe matrix. In section 3.4, the numerical method is validated with the analytical solution of the Riemann problem of nonlinear elastodynamics [22]. Section 4 introduces the modifications of the equations to account for the slow dynamics, as well as the modifications of the numerical method. Then, the propagation of a sinusoidal wave in the material is addressed (section 4.4). The results are in qualitative agreement with experimental observations. 


\section{Nonlinear elastodynamics}

\subsection{Governing equations}

Lagrangian hyperelasticity. Let us consider an homogeneous continuum in which no heat transfer occurs. Furthermore, self-gravitation is neglected. A particle initially located at some position $x_{0}$ of the reference configuration moves to a position $\boldsymbol{x}_{t}$ of the current configuration. The deformation gradient is a second-order tensor defined by (see e.g. [23, 24, 25, 26])

$$
F=\operatorname{grad} x_{t}=I+\operatorname{grad} u,
$$

where $\boldsymbol{u}=\boldsymbol{x}_{\boldsymbol{t}}-\boldsymbol{x}_{0}$ denotes the displacement field and $\boldsymbol{g} \boldsymbol{r} \boldsymbol{a d}$ is the gradient with respect to the material coordinates $\boldsymbol{x}_{0}$ (Lagrangian gradient). In the reference configuration, the deformation gradient (1) is equal to the metric tensor $\boldsymbol{I}$. Here, the Euclidean space is described by an orthonormal basis $\left(\boldsymbol{e}_{1}, \boldsymbol{e}_{2}, \boldsymbol{e}_{3}\right)$ and a Cartesian coordinate system $(O, x, y, z)$. In this case, the matrix of the coordinates of $\boldsymbol{I}$ is the identity matrix.

Here, the Lagrangian representation of motion is used. The material derivative of the deformation gradient satisfies

$$
\dot{F}=\operatorname{grad} v,
$$

where $v\left(x_{0}, t\right)$ is the velocity field. The conservation of mass implies $\rho_{0} / \rho=\operatorname{det}(\boldsymbol{F})$, where $\rho$ denotes the mass density in the deformed configuration, and $\rho_{0}$ denotes the mass density in the reference configuration. The motion is also driven by the conservation of momentum

$$
\rho_{0} \dot{\boldsymbol{v}}=\operatorname{div}\left(\operatorname{det}(\boldsymbol{F}) \boldsymbol{\sigma} \cdot \boldsymbol{F}^{-\top}\right)+\boldsymbol{f}^{v},
$$

where $\boldsymbol{d i v}$ denotes the divergence with respect to the material coordinates. The tensor $\boldsymbol{\sigma}=\boldsymbol{\sigma}^{\top}$ is the Cauchy stress tensor, and $\boldsymbol{f}^{v}$ is an external volume force applied to the material.

In hyperelasticity, the only variables of state are the specific entropy $\eta$ and a strain tensor. Moreover, the dissipation in the material is zero, i.e. the thermodynamic process is isentropic. Here, the deformation of the material is represented by the Green-Lagrange strain tensor, but other choices are possible. The Green-Lagrange strain tensor is defined as $\boldsymbol{E}=\frac{1}{2}(\boldsymbol{C}-\boldsymbol{I})$ where $\boldsymbol{C}=\boldsymbol{F}^{\top} \cdot \boldsymbol{F}$ is the right Cauchy-Green strain tensor, or equivalently as a function of the displacement gradient tensor,

$$
E=\frac{1}{2}\left(\operatorname{grad} u+\operatorname{grad}^{\top} u+\operatorname{grad}^{\top} u \cdot \operatorname{grad} u\right) .
$$

The internal energy per unit of reference volume is $W(\boldsymbol{E})$, where $W$ is the strain energy density function. Under these assumptions, the expression of the Cauchy stress tensor is

$$
\boldsymbol{\sigma}=\frac{1}{\operatorname{det}(\boldsymbol{F})} \boldsymbol{F} \cdot \frac{\partial W}{\partial \boldsymbol{E}} \cdot \boldsymbol{F}^{\top}
$$

Thus, the first Piola-Kirchhoff tensor in (3) satisfies

$$
\operatorname{det}(\boldsymbol{F}) \boldsymbol{\sigma} \cdot \boldsymbol{F}^{-\top}=\boldsymbol{F} \cdot \frac{\partial W}{\partial \boldsymbol{E}} .
$$

In the isotropic case, the strain energy is a function of the invariants of $\boldsymbol{E}$ defined by

$$
\begin{aligned}
E_{\mathrm{I}} & =\operatorname{tr}(\boldsymbol{E}), \\
E_{\mathrm{II}} & =\frac{1}{2}\left(\operatorname{tr}(\boldsymbol{E})^{2}-\operatorname{tr}\left(\boldsymbol{E}^{2}\right)\right), \\
E_{\mathrm{III}} & =\operatorname{det}(\boldsymbol{E}) .
\end{aligned}
$$

The derivative of the strain energy with respect to the strain tensor writes

$$
\frac{\partial W}{\partial \boldsymbol{E}}=\alpha_{0} \boldsymbol{I}+\alpha_{1} \boldsymbol{E}+\alpha_{2} \boldsymbol{E}^{2},
$$

where

$$
\begin{aligned}
& \alpha_{0}=\frac{\partial W}{\partial E_{\mathrm{I}}}+E_{\mathrm{I}} \frac{\partial W}{\partial E_{\mathrm{II}}}+E_{\mathrm{II}} \frac{\partial W}{\partial E_{\mathrm{III}}} \\
& \alpha_{1}=-\frac{\partial W}{\partial E_{\mathrm{II}}}-E_{\mathrm{I}} \frac{\partial W}{\partial E_{\mathrm{III}}} \\
& \alpha_{2}=\frac{\partial W}{\partial E_{\mathrm{III}}}
\end{aligned}
$$

are functions of the invariants $\left(E_{\mathrm{I}}, E_{\mathrm{II}}, E_{\mathrm{III}}\right)$.

Longitudinal plane waves. We make the assumption that the displacement field $\boldsymbol{u}$ has no component along $\boldsymbol{e}_{2}$ and $\boldsymbol{e}_{3}$. Moreover, its component $u$ along $\boldsymbol{e}_{1}$ is independent on $y$ and $z$. Therefore, the displacement gradient writes $\operatorname{grad} u=\varepsilon\left(\boldsymbol{e}_{1} \otimes \boldsymbol{e}_{1}\right)$, where $\varepsilon=\partial_{x} u>-1$ is the axial component of the displacement gradient. The invariants (7) of the Green-Lagrange strain tensor are $E_{\mathrm{I}}=\varepsilon+\frac{1}{2} \varepsilon^{2}$ and $E_{\mathrm{II}}=0=E_{\mathrm{III}}$. Thus, the strain energy $W$ is now a function of $\varepsilon$ only. In the longitudinal case, the $11^{-}$ coordinate of the first Piola-Kirchhoff tensor (6) is equal to the coordinate $\sigma$ of the Cauchy stress tensor. Equations (8)-(9) and the expression of the invariants provide the stress-strain relationship

$$
\begin{aligned}
\sigma & =(1+\varepsilon)\left(\alpha_{0}+E_{\mathrm{I}} \alpha_{1}+E_{\mathrm{I}}^{2} \alpha_{2}\right) \\
& =\frac{\partial E_{\mathrm{I}}}{\partial \varepsilon} \frac{\partial W}{\partial E_{\mathrm{I}}} \\
& =W^{\prime}(\varepsilon),
\end{aligned}
$$

where the ' denotes the total derivative with respect to $\varepsilon$.

Constitutive laws. The Murnaghan model of hyperelasticity [19] is widely used in the communities of geophysics and nondestructive testing [27, 28, 20, 21]. Its strain energy density function is

$$
\begin{aligned}
W= & \frac{\lambda+2 \mu}{2} E_{\mathrm{I}}^{2}-2 \mu E_{\mathrm{II}}+\frac{\mathfrak{l}+2 \mathfrak{m}}{3} E_{\mathrm{I}}^{3} \\
& -2 \mathfrak{m} E_{\mathrm{I}} E_{\mathrm{II}}+\mathfrak{n} E_{\mathrm{III}},
\end{aligned}
$$

where $(\lambda, \mu)$ are the Lamé parameters and $(\mathfrak{l}, \mathfrak{m}, \mathfrak{n})$ are the Murnaghan coefficients. Sometimes, Landau's law with parameters $(\mathcal{A}, \mathcal{B}, \mathcal{C})$ is used instead, and the relationship with Murnaghan's law (11) is specified in [26]. With the 
above expression of the strain energy (11), the axial component of the Cauchy stress (10) is

$$
\begin{gathered}
\sigma=M_{0} \varepsilon\left(1+\left(\frac{3}{2}+\vartheta\right) \varepsilon+\left(\frac{1}{2}+2 \vartheta\right) \varepsilon^{2}\right. \\
\left.+\frac{5 \vartheta}{4} \varepsilon^{3}+\frac{\vartheta}{4} \varepsilon^{4}\right)
\end{gathered}
$$

where $M_{0}=\lambda+2 \mu>0$ is the elastic modulus, and $\vartheta=(\mathfrak{l}+2 \mathfrak{m}) /(\lambda+2 \mu)$. If the Murnaghan coefficients equal zero $(\vartheta=0)$, only geometric nonlinearities remain, and the Saint Venant-Kirchhoff model of hyperelasticity is recovered. The later reduces to the classical Hooke's law in the case of infinitesimal strain $\varepsilon \simeq 0$.

When geometric nonlinearities are neglected (e.g., when the Murnaghan coefficients are very large), the Green-Lagrange strain tensor is linearized with respect to the components of $\operatorname{grad} \boldsymbol{u}$, so that the strain tensor (4) is replaced by the infinitesimal strain tensor: $\boldsymbol{E} \simeq \boldsymbol{\varepsilon}$. Doing this, the first invariant of the strain tensor is $E_{\mathrm{I}} \simeq \varepsilon$ in the longitudinal case. Moreover, the first Piola-Kirchhoff stress is linearized with respect to the components of $\operatorname{grad} \boldsymbol{u}$ as well, i.e. $\boldsymbol{F} \cdot \partial W / \partial \boldsymbol{E} \simeq \partial W / \partial \boldsymbol{\varepsilon}$ in (6). Doing this, the longitudinal constitutive law (10) reduces to $\sigma=\alpha_{0}+\alpha_{1} \varepsilon+\alpha_{2} \varepsilon^{2}$, and (12) becomes

$$
\sigma=M_{0} \varepsilon(1+\vartheta \varepsilon)
$$

This constitutive law corresponds to a quadratic polynomial $\sigma=M_{0} \varepsilon(1-\beta \varepsilon)$, with $\beta=-\vartheta$.

A cubic polynomial constitutive law $[7,8,10]$

$$
\sigma=M_{0} \varepsilon\left(1-\beta \varepsilon-\delta \varepsilon^{2}\right)
$$

is also widely used to describe nonlinear elasticity in solids. If the geometric nonlinearities are negligible (13), then the parameters $\beta=-\vartheta$ and $\delta=0$ correspond to Murnaghan's law. If the geometric nonlinearities are taken into account (12), then the choice $-\beta=\frac{3}{2}+\vartheta$ and $-\delta=\frac{1}{2}+2 \vartheta$ makes the two models coincide up to the third order. Contrary to (12), orders 4 and 5 are not taken into account in (14), which shows that both models are not equivalent.

The major difference between the stress-strain relationships (12) and (14) is the number of independent parameters. In (14), the cubic term can be set independently of the quadratic term. In (12), the cubic term is not independent on the quadratic term: if (12) and (14) are assimilated, then $\delta=\frac{5}{2}+2 \beta$. However, experimental evidence shows that $\delta$ is larger than $\beta$ by several orders of magnitude, so that a constitutive law of the Murnaghan type is not sufficient to represent accurately elastic nonlinearity in geomaterials [3]. From now on, the cubic polynomial law (14) is used.

\subsection{Hyperbolic system of conservation laws}

The equations of motion (2)-(3) write as a non-homogeneous system of conservation laws with respect to the variables $\boldsymbol{q}=(\varepsilon, v)^{\top}$, where $v$ is the particle velocity component along $\boldsymbol{e}_{1}$,

$$
\partial_{t} \boldsymbol{q}+\partial_{x} \boldsymbol{f}(\boldsymbol{q})=\boldsymbol{s}
$$

The flux function is $\boldsymbol{f}(\boldsymbol{q})=-\left(v, \sigma(\varepsilon) / \rho_{0}\right)^{\top}$, and the forcing is $s=\left(0, f^{v} / \rho_{0}\right)^{\top}$, where $f^{v}$ is the volume force component along $\boldsymbol{e}_{1}$. The Jacobian matrix of the flux is

$$
f^{\prime}(q)=-\left(\begin{array}{cc}
0 & 1 \\
\sigma^{\prime}(\varepsilon) / \rho_{0} & 0
\end{array}\right)
$$

where $\sigma^{\prime}=W^{\prime \prime}$ is the derivative of $\sigma$ with respect to $\varepsilon$. The eigenvalues of $\boldsymbol{f}^{\prime}(\boldsymbol{q})$ are $\{-c(\boldsymbol{q}), c(\boldsymbol{q})\}$, where $c(\boldsymbol{q})=$ $\sqrt{\sigma^{\prime}(\varepsilon) / \rho_{0}}$ is the speed of sound. The system of conservation laws is strictly hyperbolic if $\sigma^{\prime}(\varepsilon)>0$, i.e. over a domain where the strain energy $W$ is a strictly convex function of $\varepsilon$. Some properties of the system (15) are listed below without proof. Interested readers are referred to standard textbooks for more details about hyperbolic systems $[16,17]$.

In the case of the cubic law (14), strict hyperbolicity is ensured if [22]

$$
\varepsilon \in] \frac{1}{\beta-\sqrt{\beta^{2}+3 \delta}}, \frac{1}{\beta+\sqrt{\beta^{2}+3 \delta}}[.
$$

If $\beta=\delta=0$, then the polynomial law (14) amounts to Hooke's law, and the characteristic fields are linearly degenerate. If $\beta \neq 0$ and $\delta=0$, then the polynomial law is quadratic, and the stress is either a strictly convex or a strictly concave function of the strain. The characteristic fields are genuinely nonlinear. If $\delta \neq 0$, then the polynomial law (14) is neither convex nor concave. Indeed, an inflexion point is located at $\varepsilon_{0}=-\beta / 3 \delta$. The characteristic fields are neither genuinely nonlinear nor linearly degenerate (i.e., they are nongenuinely nonlinear). In the case of Murnaghan's law (12), a similar analysis is carried out in the appendix.

\section{Finite volumes with flux limiters}

\subsection{Conservative scheme}

In the examples presented later on, the physical domain is unbounded. We consider a finite numerical domain $\left[x_{0}, x_{N}\right]$. It is discretized using a regular grid in space with step $\Delta x=\left(x_{N}-x_{0}\right) / N$. Also, a variable time step $\Delta t=t_{n+1}-t_{n}$ is introduced. Therefore, $q\left(x_{i}, t_{n}\right)$ denotes the solution to (15) at the abscissa $x_{i}=x_{0}+i \Delta x$ and at the time $t_{n}$. The volume force $f^{v}$ is assumed to be a point load $f^{v}=\delta_{s}(x) \varphi(t)$, where $\delta_{s}(x)=\delta\left(x-x_{s}\right)$ is the Dirac delta located at the abscissa $x=x_{s}$, and $\varphi(t)$ is the source signal.

The non-homogeneous system of conservation laws (15) is integrated explicitly:

$$
q_{i}^{n+1}=q_{i}^{n}-\frac{\Delta t}{\Delta x}\left(f_{i+1 / 2}^{n}-f_{i-1 / 2}^{n}\right)+\frac{\Delta t}{\Delta x} s_{i}^{n},
$$


where $\boldsymbol{q}_{i}^{n} \simeq \boldsymbol{q}\left(x_{i}, t_{n}\right)$ approximates the solution at the grid nodes, and $s_{i}^{n}=s\left(x_{i}, t_{n}\right)$. The numerical flux $\boldsymbol{f}_{i+1 / 2}^{n}$ of the flux-limiter method is specified later on. This scheme is stable under the classical CFL condition

$$
\kappa=\frac{\Delta t}{\Delta x} c_{\max }^{n} \leqslant 1
$$

where $\kappa$ is the Courant number, and $c_{\max }^{n}$ is the maximum sound speed that is encountered at time $t_{n}$. If $\sigma$ is convex or concave, then the maximum sound speed at time $t_{n}$ is

$$
c_{\max }^{n}=\max _{0 \leqslant i \leqslant N} c\left(\boldsymbol{q}_{i}^{n}\right)
$$

If the constitutive law is neither convex nor concave, sound speeds larger than (20) may be reached (see e.g. section 16.1 in [17]). The local maximum sound speed is obtained by maximizing $c(\boldsymbol{q})$ for $\boldsymbol{q}$ between $\boldsymbol{q}_{i}^{n}$ and $\boldsymbol{q}_{i+1}^{n}$. Then, one obtains

$$
c_{\max }^{n}=\max _{0 \leqslant i<N} \sqrt{\frac{1}{\rho_{0}} \max _{\varepsilon \in \mathcal{D}_{i}} \sigma^{\prime}(\varepsilon)},
$$

where $\mathcal{D}_{i}$ is the interval with bounds $\varepsilon_{i}^{n}$ and $\varepsilon_{i+1}^{n}$. Finally, the method has a variable time step satisfying $\Delta t=$ $\kappa \Delta x / c_{\max }^{n}$.

Since the flux function $\boldsymbol{f}$ is nonlinear with respect to $\boldsymbol{q}$, an initial-value problem (or Cauchy problem) of the homogeneous system defined by the data $q(x, 0)$ at the time $t=0$ can have several weak solutions (i.e. solutions of an integral form of (15)). It is not straightforward for a numerical method to converge towards the correct weak solution. In particular, the numerical fluxes $f_{i+1 / 2}^{n}$ in (44) must be computed carefully. Given that the eigenvalues $\pm c(\boldsymbol{q})$ of (16) have constant sign, no transsonic rarefaction can occur. Moreover, no slow-moving shock can occur either, due to the monotonicity of $\sigma$ over the domain of hyperbolicity (17). Therefore, Roe linearization can be used to construct an accurate numerical scheme.

\subsection{Roe linearization}

The nonlinear flux $\boldsymbol{f}(\boldsymbol{q})$ is approximated locally by the linear flux $\boldsymbol{A}_{i+1 / 2} \boldsymbol{q}$. A Roe matrix $\boldsymbol{A}_{i+1 / 2}$ approximates the Jacobian $\boldsymbol{f}^{\prime}\left(\boldsymbol{q}_{i+1 / 2}^{n}\right)$ at the midpoint of $\left[x_{i}, x_{i+1}\right]$ and the time $t_{n}$, in such a way that

1. $\boldsymbol{A}_{i+1 / 2}$ is diagonalizable with real eigenvalues;

2. $\boldsymbol{A}_{i+1 / 2}\left(\boldsymbol{q}_{i+1}^{n}-\boldsymbol{q}_{i}^{n}\right)=\boldsymbol{f}\left(\boldsymbol{q}_{i+1}^{n}\right)-\boldsymbol{f}\left(\boldsymbol{q}_{i}^{n}\right)$;

3. $\lim _{\boldsymbol{q}_{i+1}^{n} \rightarrow \boldsymbol{q}_{i}^{n}} \boldsymbol{A}_{i+1 / 2}=\boldsymbol{f}^{\prime}\left(\boldsymbol{q}_{i}^{n}\right)$.

According to section 15.3.2 of [17], such a matrix may be expressed by the formula

$$
\boldsymbol{A}_{i+1 / 2}=\int_{0}^{1} \boldsymbol{f}^{\prime}\left(\boldsymbol{q}_{i}^{n}+\xi\left(\boldsymbol{q}_{i+1}^{n}-\boldsymbol{q}_{i}^{n}\right)\right) \mathrm{d} \xi
$$

which ensures that the properties 2. and 3. are satisfied.
In the case of the system (15), which corresponds to exercise 15.1.(a) p. 349 of [17], the following matrix is obtained:

$$
\boldsymbol{A}_{i+1 / 2}=\left(\begin{array}{cc}
0 & -1 \\
-a_{i+1 / 2} & 0
\end{array}\right),
$$

where

$$
a_{i+1 / 2}=\frac{W^{\prime}\left(\varepsilon_{i+1}^{n}\right)-W^{\prime}\left(\varepsilon_{i}^{n}\right)}{\rho_{0}\left(\varepsilon_{i+1}^{n}-\varepsilon_{i}^{n}\right)} .
$$

To avoid divisions by zero when $\varepsilon_{i}^{n}=\varepsilon_{i+1}^{n}$, one computes

$$
a_{i+1 / 2}=\frac{W^{\prime \prime}\left(\varepsilon_{i}^{n}\right)}{\rho_{0}}
$$

in this particular case. The eigenvalues of the matrix (23) are $\left\{-s_{i+1 / 2}, s_{i+1 / 2}\right\}$, where $s_{i+1 / 2}=\sqrt{a_{i+1 / 2}}$. Since the strain energy function $W$ is convex over the hyperbolicity domain, the coefficient $a_{i+1 / 2}$ is positive. Therefore, the eigenvalue $s_{i+1 / 2}$ is real, and the property 1 . is satisfied. The matrix (47) is a Roe matrix.

For later use, we introduce the decomposition of $\boldsymbol{q}_{i+1}^{n}-$ $\boldsymbol{q}_{i}^{n}$ in the basis of right eigenvectors of the Roe matrix

$$
\begin{aligned}
& \boldsymbol{p}_{i+1 / 2}^{1}=\left(1, s_{i+1 / 2}\right)^{\top}, \\
& \boldsymbol{p}_{i+1 / 2}^{2}=\left(1,-s_{i+1 / 2}\right)^{\top} .
\end{aligned}
$$

The jump of the solution can be expanded as

$$
\begin{aligned}
\boldsymbol{q}_{i+1}^{n}-\boldsymbol{q}_{i}^{n} & =\sum_{k=1}^{2} \alpha_{i+1 / 2}^{k} \boldsymbol{p}_{i+1 / 2}^{k} \\
& =\sum_{k=1}^{2} \boldsymbol{W}_{i+1 / 2}^{k}
\end{aligned}
$$

with the coefficients

$$
\begin{aligned}
& \alpha_{i+1 / 2}^{1}=\frac{1}{2}\left(\varepsilon_{i+1}^{n}-\varepsilon_{i}^{n}+\frac{v_{i+1}^{n}-v_{i}^{n}}{s_{i+1 / 2}}\right), \\
& \alpha_{i+1 / 2}^{2}=\frac{1}{2}\left(\varepsilon_{i+1}^{n}-\varepsilon_{i}^{n}-\frac{v_{i+1}^{n}-v_{i}^{n}}{s_{i+1 / 2}}\right) .
\end{aligned}
$$

\subsection{Flux limiter}

We describe now the flux-limiter scheme. The numerical flux in (18) takes the form (section 15.4 in [17])

$$
f_{i+1 / 2}^{n}=f_{i+1 / 2}^{L}+f_{i+1 / 2}^{H},
$$

where $\boldsymbol{f}_{i+1 / 2}^{L}$ is the flux of Godunov's method or one of its approximated versions, and $\boldsymbol{f}_{i+1 / 2}^{H}$ is a higher-order correction deduced from the Lax-Wendroff method. Here, the Godunov flux is approximated linearly by the classical Roe flux, and the Roe matrix (47) is used. Thus,

$$
\begin{aligned}
f_{i+1 / 2}^{L}= & \frac{1}{2}\left(\boldsymbol{f}\left(\boldsymbol{q}_{i}^{n}\right)+\boldsymbol{f}\left(\boldsymbol{q}_{i+1}^{n}\right)\right) \\
& -\frac{1}{2} s_{i+1 / 2}\left(\boldsymbol{W}_{i+1 / 2}^{1}+\boldsymbol{W}_{i+1 / 2}^{2}\right),
\end{aligned}
$$


Table I. Physical parameters of Berea sandstone.

\begin{tabular}{|cccccc|}
\hline$\rho_{0}\left(\mathrm{~kg} / \mathrm{m}^{3}\right)$ & $M_{0}(\mathrm{GPa})$ & $\beta$ & $\delta$ & $\gamma\left(\mathrm{J} / \mathrm{m}^{3}\right)$ & $\tau(\mathrm{ms})$ \\
$2.2 \cdot 10^{3}$ & 14 & 50 & $10^{8}$ & 1.0 & 10 \\
\hline
\end{tabular}

where $\boldsymbol{W}_{i+1 / 2}^{k}$ is defined in (27). The higher-order correction of the flux-limiter method writes

$$
\begin{aligned}
& f_{i+1 / 2}^{H}=\frac{1}{2} s_{i+1 / 2}\left(1-\frac{\Delta t}{\Delta x} s_{i+1 / 2}\right) \\
& \cdot\left(\phi\left(\theta_{i+1 / 2}^{1}\right) \mathcal{W}_{i+1 / 2}^{1}+\phi\left(\theta_{i+1 / 2}^{2}\right) \mathcal{W}_{i+1 / 2}^{2}\right),
\end{aligned}
$$

where

$$
\begin{aligned}
& \theta_{i+1 / 2}^{1}=\frac{\mathcal{W}_{i+3 / 2}^{1} \cdot \mathcal{W}_{i+1 / 2}^{1}}{\mathcal{W}_{i+1 / 2}^{1} \cdot \mathcal{W}_{i+1 / 2}^{1}} \\
& \theta_{i+1 / 2}^{2}=\frac{\mathcal{W}_{i-1 / 2}^{2} \cdot \mathcal{W}_{i+1 / 2}^{2}}{\mathcal{W}_{i+1 / 2}^{2} \cdot \mathcal{W}_{i+1 / 2}^{2}}
\end{aligned}
$$

and $\phi$ is a limiter function. Here, the minmod limiter

$$
\phi(\theta)=\max \{0, \min \{1, \theta\}\}
$$

is used.

The weights $\phi\left(\theta_{i+1 / 2}^{k}\right)$ are designed to avoid spurious oscillations in the numerical solution. If the weights $\phi\left(\theta_{i+1 / 2}^{k}\right)$ equal one in (31), then the Lax-Wendroff method based on the Roe matrix (47) is recovered. In the case of nonconvex flux functions, the minmod limiter (33) has shown better convergence properties than other limiter functions. Similar observations are reported in [29, 30].

To carry out one iteration in time at some grid node $i$, the numerical values of $\boldsymbol{q}$ at the grid nodes $i-2, \ldots, i+2$ are required. Therefore, two "ghost cells" must be added on the left and on the right of the numerical domain. Here, a zero-order extrapolation of the numerical solution can be used to achieve outflow boundary conditions. Thus, we simply set

$$
\begin{aligned}
& \boldsymbol{q}_{-2}^{n}=\boldsymbol{q}_{-1}^{n}=\boldsymbol{q}_{0}^{n}, \\
& \boldsymbol{q}_{N+2}^{n}=\boldsymbol{q}_{N+1}^{n}=\boldsymbol{q}_{N}^{n},
\end{aligned}
$$

at each time step [17].

\subsection{Numerical test case}

The orders of magnitude of the elastic parameters for Berea sandstone $\rho_{0}, M_{0}, \beta, \delta$ in table I have been taken in Table I and Figure 5a of [3]. From (17), it follows that the model (15) is hyperbolic if $|\varepsilon|<5.7 \cdot 10^{-5}$. The numerical domain is $\left[x_{0}, x_{N}\right]=[-0.5,0.5] \mathrm{m}$. To avoid instability, the Courant number (19) is set to $\kappa=0.95$.

This test is carried out to validate the ability of the numerical scheme to represent accurately nonsmooth solutions. Here, no volume force is applied in the material. We consider piecewise constant initial data with a single discontinuity at the abscissa $x=0$, i.e. a Riemann problem.
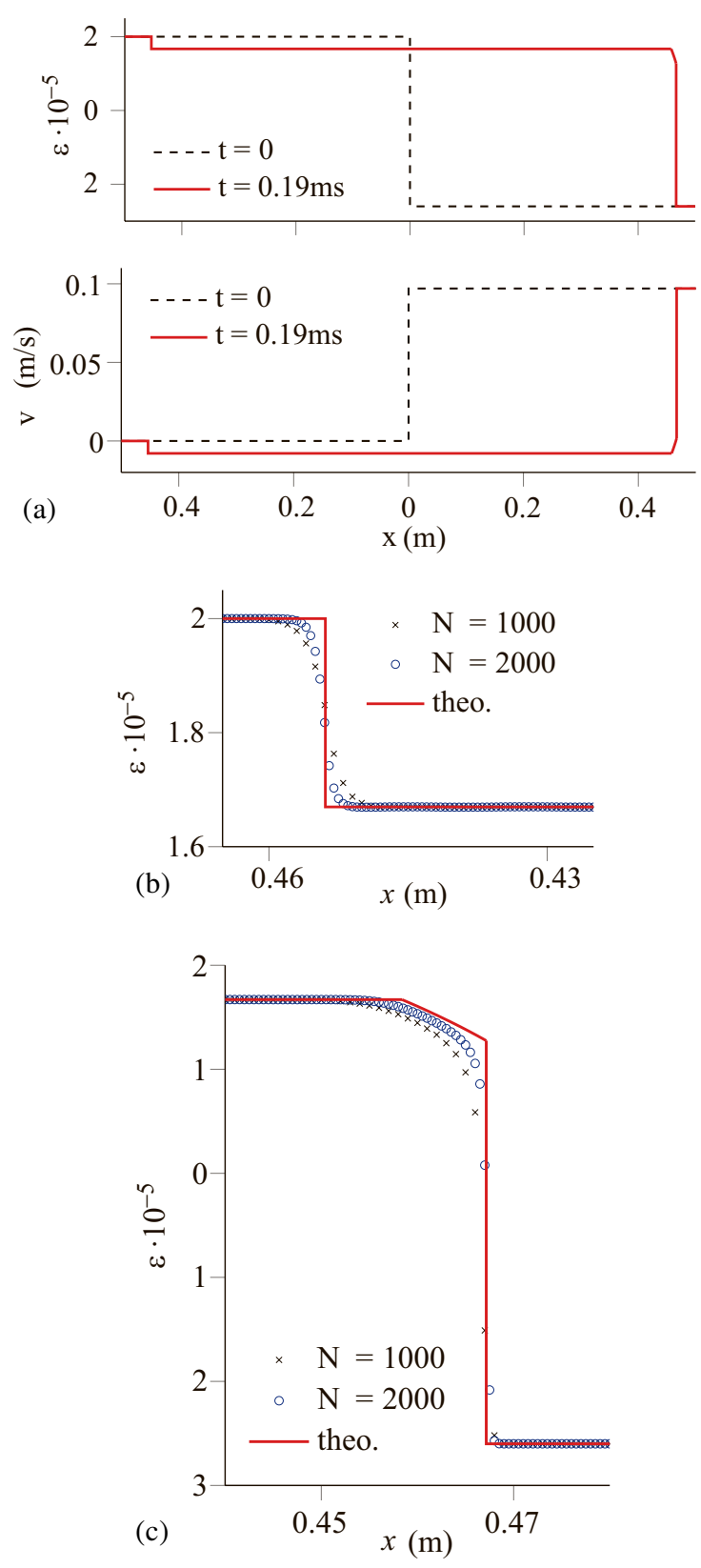

Figure 1. (a) Analytical solution to a Riemann problem. Comparison with the numerical solution (b) on the left-going strain wave, (c) and on the right-going strain wave.

Here, the initial data is $\varepsilon=2 \cdot 10^{-5}, v=0$ if $x<0$, and $\varepsilon=-2.6 \cdot 10^{-5}, v=0.097 \mathrm{~m} / \mathrm{s}$ if $x>0$. The analytical solution to this initial-value problem is displayed in Figure 1a. Details about its computation can be found in [22]. Moreover, an interactive application and a Matlab toolbox [31] can be found at http://gchiavassa.perso.centralemarseille.fr/RiemannElasto/.

The numerical solution (44) is computed up to $t=$ $0.19 \mathrm{~ms}$ on a grid with $N$ points. Figures $1 \mathrm{~b}$ and $1 \mathrm{c}$ compare the analytical solution with the numerical solution. On Figure 1b, the left-going discontinuity is represented (shock wave). On Figure 1c, the right-going compound 
wave with a continuous part followed by a discontinuous part is represented (rarefaction shock wave). Both waves are well-located and accurately computed by the numerical scheme. In particular, no spurious oscillations arise, and no convergence failure is noticed as $N$ increases from 1000 to 2000 .

\section{Coupling with slow dynamics}

As specified in the introduction, nonlinear elastodynamics presented in Section 2 is not sufficient to describe the softening of the material. In [13], a simple approach was proposed to account for such phenomena. The model was presented in 3D, and analytical computations were carried out in the longitudinal case. Here, we show how the previous numerical method is adapted to this model of slow dynamics.

\subsection{Modified equations of motion}

Internal-variable model. An internal-variable $g$ in $[0,1[$ is introduced, to represent the softening of the material. The internal energy per unit of reference volume $\rho_{0} e=$ $W(\varepsilon)$ becomes [13]

$$
\rho_{0} e=(1-g) W(\varepsilon)+\Phi(g),
$$

where $e$ is the specific internal energy. In (35), $\Phi(g)$ represents a storage energy with expression

$$
\Phi(g)=-\frac{1}{2} \gamma \ln \left(1-g^{2}\right)
$$

where $\gamma>0$ is an energy per unit volume, but other choices are suitable. As discussed in [13], the storage energy (36) is chosen such that equilibrium points of the model are unique, $g=0$ is an equilibrium point, and $g$ is bounded by 1 . With such a choice, one observes that the internal energy per unit of reference volume (35) is equal to the strain energy $W$ when $g$ is equal to zero.

The second principle of thermodynamics yields the axial component of the Cauchy stress [13]

$$
\sigma=(1-g) W^{\prime}(\varepsilon)
$$

instead of $W^{\prime}(\varepsilon)(10)$. The simplest thermodynamically admissible choice of evolution equation is [13]

$$
\tau_{1} \dot{g}=W(\varepsilon)-\Phi^{\prime}(g)
$$

where $\tau_{1}=\tau \cdot 1 \mathrm{~J} / \mathrm{m}^{3}$ and $\tau>0$ is a time constant. With the laws (37) and (38), the dissipation per unit of reference volume is [13]

$$
\rho_{0} T \dot{\eta}=-\rho_{0} \frac{\partial e}{\partial g} \dot{g}=\tau_{1}(\dot{g})^{2} \geqslant 0,
$$

where $T>0$ is the absolute temperature, and $\eta$ is the specific entropy.
Conservation laws with relaxation. The variable $g$ is added to the vector of unknowns $\boldsymbol{q}$, so that $\boldsymbol{q}=(\varepsilon, v, g)^{\top}$. The system of conservation laws (15) becomes

$$
\partial_{t} \boldsymbol{q}+\partial_{x} \boldsymbol{f}(\boldsymbol{q})=\boldsymbol{r}(\boldsymbol{q})+\boldsymbol{s},
$$

where

$$
\begin{aligned}
\boldsymbol{f}(\boldsymbol{q}) & =-\left(v,(1-g) W^{\prime}(\varepsilon) / \rho_{0}, 0\right)^{\top}, \\
\boldsymbol{r}(\boldsymbol{q}) & =\left(0,0,\left(W(\varepsilon)-\Phi^{\prime}(g)\right) / \tau_{1}\right)^{\top}, \\
\boldsymbol{s} & =\left(0, f_{v} / \rho_{0}, 0\right)^{\top} .
\end{aligned}
$$

The Jacobian matrix $\boldsymbol{f}^{\prime}(\boldsymbol{q})$ of $\boldsymbol{f}$ has the eigenvalues $\{-c(\boldsymbol{q}), c(\boldsymbol{q}), 0\}$, where

$$
c(\boldsymbol{q})=\sqrt{\frac{(1-g) W^{\prime \prime}(\varepsilon)}{\rho_{0}}}
$$

is the speed of sound. The speed of sound (42) is real and nonzero - in other words, the system (40) is strictly hyperbolic — provided that $g<1$ and $W^{\prime \prime}(\varepsilon)>0$. This second condition was already required in the elastic case (15).

Now let us examine the spectrum of the relaxation function in (40). The Jacobian matrix $\boldsymbol{r}^{\prime}(\boldsymbol{q})$ of $\boldsymbol{r}$ has the eigenvalues $\left\{0,0,-\Phi^{\prime \prime}(g) / \tau_{1}\right\}$. The expression (36) ensures that $\boldsymbol{r}^{\prime}(\boldsymbol{q})$ is negative semi-definite. Its spectral radius is

$$
Y=\frac{\gamma}{\tau_{1}} \frac{1+g^{2}}{\left(1-g^{2}\right)^{2}}
$$

which involves the relaxation time $\tau_{1} / \gamma$ characteristic of the slow dynamics [13].

\subsection{Modified numerical method}

Conservative scheme. Applying the same explicit discretization as (18) to (40) yields

$$
\begin{aligned}
\boldsymbol{q}_{i}^{n+1}= & \boldsymbol{q}_{i}^{n}-\frac{\Delta t}{\Delta x}\left(f_{i+1 / 2}^{n}-f_{i-1 / 2}^{n}\right) \\
& +\Delta t \boldsymbol{r}\left(q_{i}^{n}\right)+\frac{\Delta t}{\Delta x} s_{i}^{n},
\end{aligned}
$$

where the numerical flux $\boldsymbol{f}_{i+1 / 2}^{n}$ is specified later on. According to the time-marching formula (44), explicit singlestep time integration is used.

Numerical stability imposes a bound of the form

$$
\Delta t \leqslant \min \left\{\frac{\Delta x}{c_{\max }^{n}}, \frac{2}{Y_{\max }^{n}}\right\},
$$

where $c_{\max }^{n}$ is the maximum sound velocity (42) that is encountered at time $t_{n}$, and $Y_{\max }^{n}$ is the maximum spectral radius of the relaxation function (43). Since the relaxation time of the slow dynamics is much larger than the period of exciting signals, the condition (45) reduces to the classical CFL condition (19). If the constitutive law $\varepsilon \mapsto W^{\prime}(\varepsilon)$ is convex or concave, then the maximum sound speed at time $t_{n}$ is given by (20), with the sound speed (42). If the constitutive law is neither convex nor concave, then (21) becomes

$$
c_{\text {max }}^{n}=\max _{0 \leqslant i<N} \sqrt{\frac{1-\min \left\{g_{i}^{n}, g_{i+1}^{n}\right\}}{\rho_{0}} \max _{\varepsilon \in \mathcal{D}_{i}} W^{\prime \prime}(\varepsilon)},
$$

where $\mathcal{D}_{i}$ is the interval with bounds $\varepsilon_{i}^{n}$ and $\varepsilon_{i+1}^{n}$. 
Roe linearization. In the case of the system (40), the following matrix is obtained from (22):

$$
\boldsymbol{A}_{i+1 / 2}=\left(\begin{array}{ccc}
0 & -1 & 0 \\
-a_{i+1 / 2} & 0 & b_{i+1 / 2} \\
0 & 0 & 0
\end{array}\right)
$$

where

$$
\begin{aligned}
a_{i+1 / 2} & =\frac{\sigma_{i+1}^{n}-\sigma_{i}^{n}}{\rho_{0}\left(\varepsilon_{i+1}^{n}-\varepsilon_{i}^{n}\right)}+\frac{g_{i+1}^{n}-g_{i}^{n}}{\varepsilon_{i+1}^{n}-\varepsilon_{i}^{n}} b_{i+1 / 2}, \\
b_{i+1 / 2} & =\frac{W\left(\varepsilon_{i+1}^{n}\right)-W\left(\varepsilon_{i}^{n}\right)}{\rho_{0}\left(\varepsilon_{i+1}^{n}-\varepsilon_{i}^{n}\right)}
\end{aligned}
$$

and the stresses (37) are $\sigma_{i}^{n}=\left(1-g_{i}^{n}\right) W^{\prime}\left(\varepsilon_{i}^{n}\right)$. To avoid divisions by zero when $\varepsilon_{i}^{n}=\varepsilon_{i+1}^{n}$, one computes

$$
\begin{aligned}
& a_{i+1 / 2}=\left(1-\frac{g_{i}^{n}+g_{i+1}^{n}}{2}\right) \frac{W^{\prime \prime}\left(\varepsilon_{i}^{n}\right)}{\rho_{0}}, \\
& b_{i+1 / 2}=\frac{W^{\prime}\left(\varepsilon_{i}^{n}\right)}{\rho_{0}}
\end{aligned}
$$

in this particular case.

The eigenvalues of the matrix (47) with the coefficients (48)-(49) are $\left\{-s_{i+1 / 2}, s_{i+1 / 2}, 0\right\}$, where the expression $s_{i+1 / 2}=\sqrt{a_{i+1 / 2}}$ is unchanged. We rewrite the coefficient $a_{i+1 / 2}$ from (48) as

$$
\begin{aligned}
& a_{i+1 / 2}=\frac{W^{\prime}\left(\varepsilon_{i+1}^{n}\right)-W^{\prime}\left(\varepsilon_{i}^{n}\right)}{\rho_{0}\left(\varepsilon_{i+1}^{n}-\varepsilon_{i}^{n}\right)} \\
& -g_{i}^{n} \frac{W\left(\varepsilon_{i+1}^{n}\right)-W\left(\varepsilon_{i}^{n}\right)-W^{\prime}\left(\varepsilon_{i}^{n}\right)\left(\varepsilon_{i+1}^{n}-\varepsilon_{i}^{n}\right)}{\rho_{0}\left(\varepsilon_{i+1}^{n}-\varepsilon_{i}^{n}\right)^{2}} \\
& -g_{i+1}^{n} \frac{W\left(\varepsilon_{i}^{n}\right)-W\left(\varepsilon_{i+1}^{n}\right)-W^{\prime}\left(\varepsilon_{i+1}^{n}\right)\left(\varepsilon_{i}^{n}-\varepsilon_{i+1}^{n}\right)}{\rho_{0}\left(\varepsilon_{i+1}^{n}-\varepsilon_{i}^{n}\right)^{2}} .
\end{aligned}
$$

One can note that the first term in (50) corresponds to the elastic case (24). Moreover, the next terms vanish when $g_{i}^{n}=0=g_{i+1}^{n}$, i.e. when no softening occurs. Since the strain energy function $W$ is convex and $g<1$ over the hyperbolicity domain, the coefficient $a_{i+1 / 2}$ in (50) is positive. Finally, the eigenvalue $s_{i+1 / 2}$ is real, and the property 1 . is satisfied. The matrix (47) is a Roe matrix for (40).

Now, the jump $\boldsymbol{q}_{i+1}^{n}-\boldsymbol{q}_{i}^{n}$ is decomposed in the basis of right eigenvectors of the Roe matrix (47)

$$
\begin{aligned}
& \boldsymbol{p}_{i+1 / 2}^{1}=\left(1, s_{i+1 / 2}, 0\right)^{\top}, \\
& \boldsymbol{p}_{i+1 / 2}^{2}=\left(1,-s_{i+1 / 2}, 0\right)^{\top}, \\
& \boldsymbol{p}_{i+1 / 2}^{3}=\left(1,0, \frac{a_{i+1 / 2}}{b_{i+1 / 2}}\right)^{\top}
\end{aligned}
$$

The jump of the solution can be expanded as a sum (27) of three $\mathcal{W}_{i+1 / 2}^{k}=\alpha_{i+1 / 2}^{k} p_{i+1 / 2}^{k}$, where the coefficients are

$$
\begin{aligned}
& \alpha_{i+1 / 2}^{1}=\frac{1}{2}\left(\frac{v_{i+1}^{n}-v_{i}^{n}}{s_{i+1 / 2}}+\frac{\sigma_{i+1}^{n}-\sigma_{i}^{n}}{\rho_{0} s_{i+1 / 2}^{2}}\right), \\
& \alpha_{i+1 / 2}^{2}=\frac{1}{2}\left(-\frac{v_{i+1}^{n}-v_{i}^{n}}{s_{i+1 / 2}}+\frac{\sigma_{i+1}^{n}-\sigma_{i}^{n}}{\rho_{0} s_{i+1 / 2}}\right),
\end{aligned}
$$

$$
\alpha_{i+1 / 2}^{3}=\varepsilon_{i+1}^{n}-\varepsilon_{i}^{n}-\frac{\sigma_{i+1}^{n}-\sigma_{i}^{n}}{\rho_{0} s_{i+1 / 2}^{2}} .
$$

Since the eigenvalue corresponding to $k=3$ in the decomposition of the jump is zero, $\boldsymbol{W}_{i+1 / 2}^{3}$ does not appear in the numerical flux (29) of the flux-limiter method. Therefore, the formulas (29) to (33) can be applied without modification with the flux function (41), the eigenvalue $s_{i+1 / 2}$ deduced from (48)-(49), and the decomposition of the jump (51)-(52).

\subsection{Pulse propagation}

Figure 3 of [3] provides an order of magnitude of the characteristic time of the slow dynamics, and an order of magnitude of the average softening $\langle\Delta M / M\rangle$ for a given strain amplitude $V$. According to [13], the characteristic time of the slow dynamics is $\tau_{1} / \gamma$, and the average softening satisfies $\langle\Delta M / M\rangle \simeq-\frac{1}{4}\left(M_{0} / \gamma+6 \delta\right) V^{2}$. If this scaling rule is combined with the values in [3], then a negative value of $\gamma$ is obtained, which is not reliable. Thus, the parameters $\gamma$ and $\tau$ in table I are not chosen to reach quantitative agreement with [3], but rather to obtain comparable orders of magnitude for the characteristics of softening.

The numerical domain and the Courant number are the same as in section 3.4, but here, the softening is taken into account. The initial data is zero, and a point load $f^{v}=\delta_{s}(x) \varphi(t)$ located at the abscissa $x_{s}=0$ generates a smooth pulse with angular frequency $\omega_{c}=2 \pi f_{c}$, with $f_{c}=6 \mathrm{kHz}$. The source signal in (40) satisfies

$$
\varphi(t)=2 M_{0} V \sum_{m=1}^{4} a_{m} \sin \left(2^{m-1} \omega_{c} t\right)
$$

where $V=6.64 \cdot 10^{-6}, a_{1}=1, a_{2}=-21 / 32, a_{3}=$ $63 / 768$, and $a_{4}=-1 / 512$. It is turned on from $t=0$ to $t=1 / f_{c}$, which corresponds to one fundamental period. Chosen for the smoothness of its time-evolution (53), the point source generates left-going and right-going waves with strain amplitude $1.507 \mathrm{~V} \approx 10^{-5}$.

The reference solution is an oversampled numerical solution (44) computed at $t=0.19 \mathrm{~ms}$ on a grid with $N^{\text {ref }}=2^{15}$ points (Figure $2 \mathrm{a}$ ). The pulse injected at $x_{s}=0$ has propagated towards both increasing and decreasing $x$. By symmetry, only the right-going part is displayed here. No shock wave is observed: the waveform is slightly distorted but keeps smooth. Figure $2 b$ illustrates the convergence of the numerical method. For a given coarse spatial discretization where $N \ll N^{\text {ref }}$, the numerical solution is 


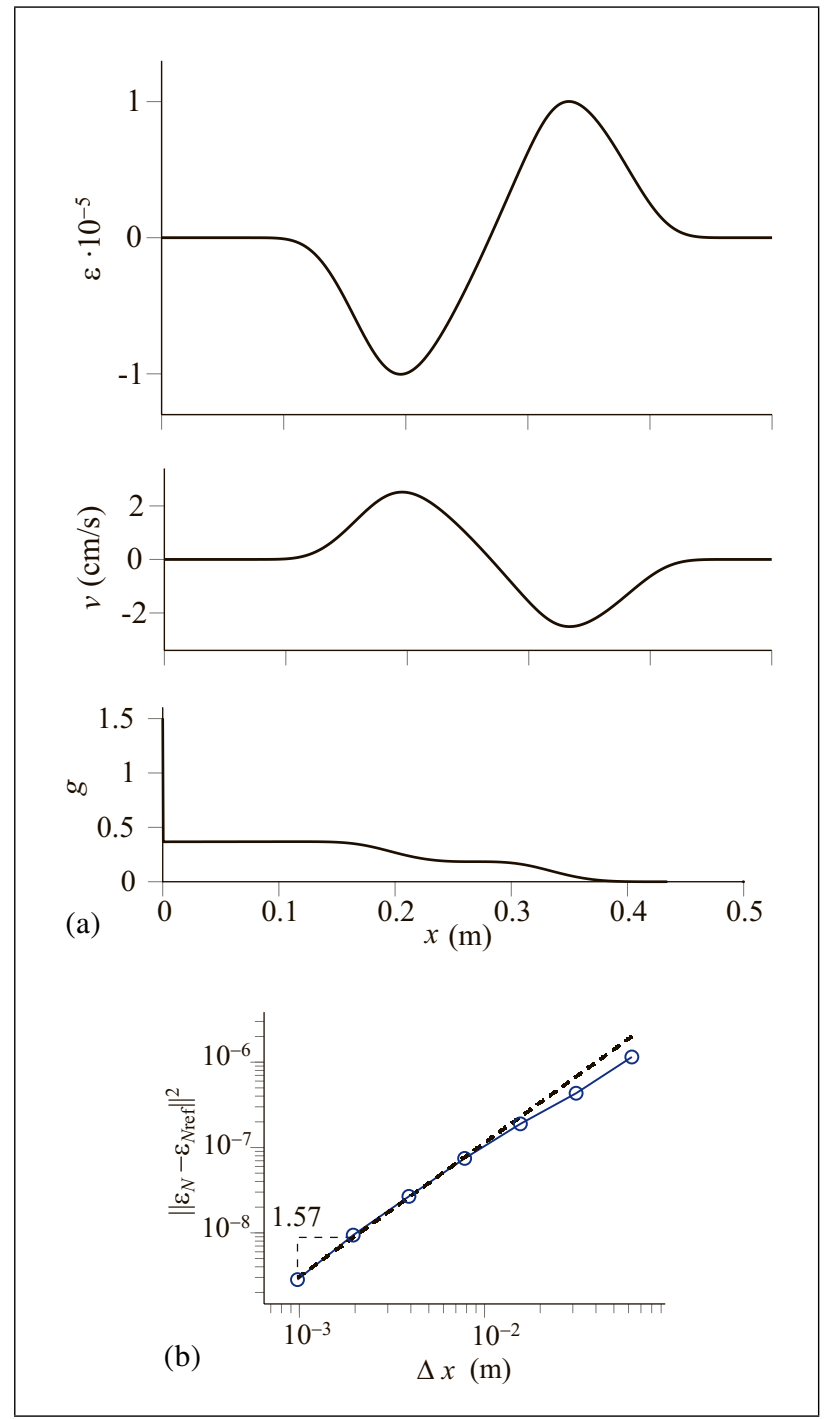

Figure 2. Assessment of the numerical method's convergence on the propagation of a smooth pulse. (a) Reference solution at $t=$ $0.19 \mathrm{~ms}$ : strain, particle velocity, and softening variable. (b) Error measurement.

computed up to $t \approx 0.19 \mathrm{~ms}$, and is compared to the reference numerical solution at the same final time. The evolution of the $L^{2}$ global error between both strain waveforms is represented in Figure $2 \mathrm{~b}$ with respect to $\Delta x=1 / N$. One can observe that the order of convergence is between one and two.

\subsection{Dynamic acousto-elasticity}

The setup is the same as in the previous section, but here, the point load generates a sinusoidal strain with amplitude $V \approx 10^{-6}$ and angular frequency $\omega_{c}=2 \pi f_{c}$, with $f_{c}=$ $5 \mathrm{kHz}$ :

$$
\varphi(t)=2 M_{0} V \sin \left(\omega_{c} t\right) .
$$

The source (54) is turned on from $t=0$ to $t=40 \mathrm{~ms}$, which corresponds to 200 periods of signal. A receiver records the numerical solution at the abscissa $x_{r}=0.3 \mathrm{~m}$.

The numerical solution (44) is computed up to $t=$ $80 \mathrm{~ms}$ on a grid with $N=80$ points, which corresponds
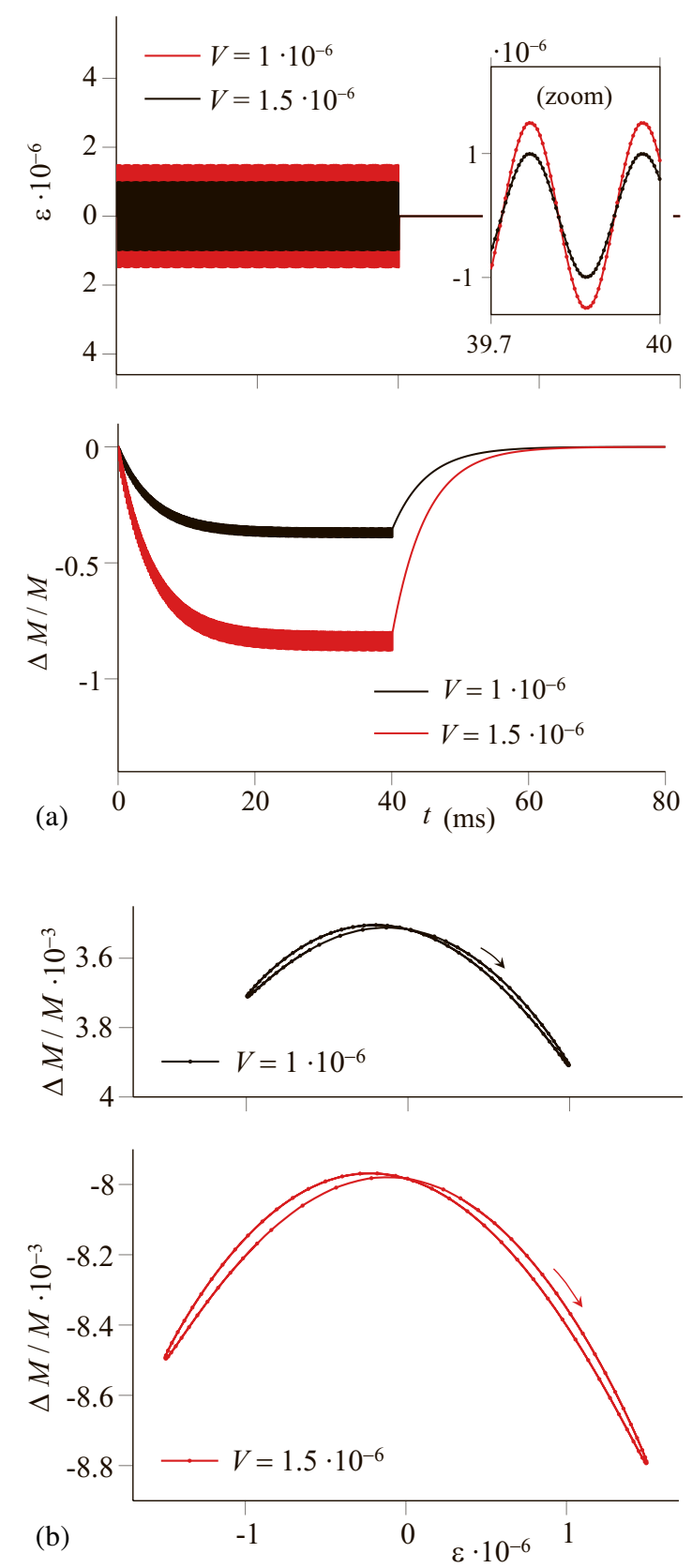

Figure 3. (Colour online) (a) Strain signal and softening of the material, as recorded by the receiver at the abscissa $x_{r}=0.3 \mathrm{~m}$. (b) Hysteresis curves in steady-state. The arrow indicates how time increases along the curve.

to 40 points per wavelength at the angular frequency $\omega_{c}$. The computations are performed in $\mathrm{C}++$. Each simulation lasts around $1.5 \mathrm{~s}$, when a recent desktop computer is used (Intel Core i5-4690, 3.5 GHz, 16 Go, 2015). Figure 3a displays the strain $\varepsilon$ and the variation of the elastic modulus

$$
\frac{\Delta M}{M}=\frac{\rho_{0} c^{2}-M_{0}}{M_{0}}
$$

deduced from the sound speed (42), which are recorded at the position $x_{r}$ of the receiver. A slow decrease of the elastic modulus combined with fast oscillations is observed 


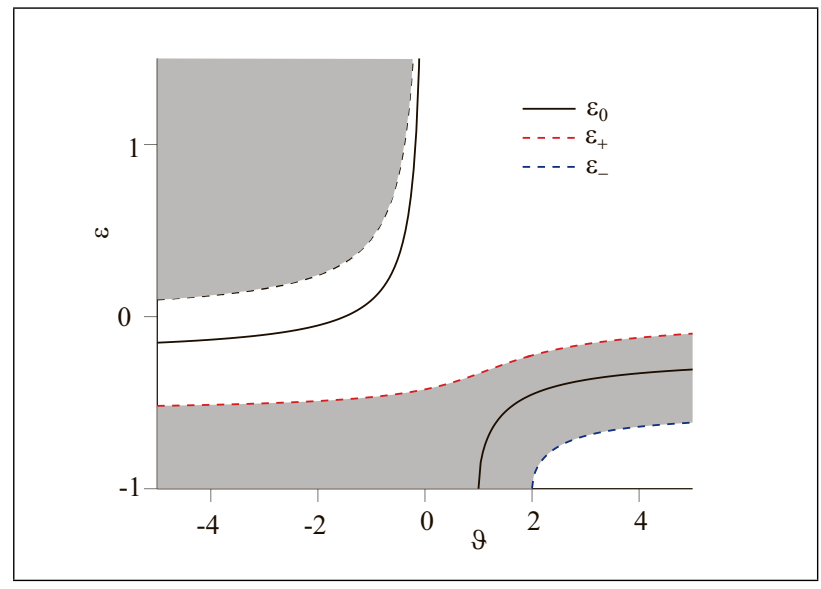

Figure 4. (Colour online) Sketch of the hyperbolicity domains (white) of Murnaghan's law (12) with respect to the parameter $\vartheta=(\mathfrak{l}+2 \mathfrak{m}) /(\lambda+2 \mu)$.

until the source is stopped. Simultaneously, the strain signals are smooth sinusoids, so that $N=80$ is sufficient. After the source is stopped, the elastic modulus recovers slowly its initial value, while the strain is equal to zero.

Figure $3 b$ focuses on the steady-state solution. Here, the last 80 numerical values before $t=40 \mathrm{~ms}$ are used, which corresponds to two periods of signal at the frequency $f_{c}$. When $\Delta M / M$ from (55) is represented with respect to the strain recorded at the position of the receiver $x_{r}$, a hysteresis curve is obtained. The orders of magnitude of these phenomena - duration of the transients, magnitude of the softening, size and shape of the hysteresis curves are very similar to those reported in [3] for Berea sandstone. In particular, one can note that the average softening $\langle\Delta M / M\rangle$ is proportional to $V^{2}$, as predicted in [13]. However, only qualitative agreement with Figure 5a of [3] is obtained.

\section{Conclusion}

A finite-volume method with flux-limiters for nonlinear longitudinal elastodynamics is implemented, with various polynomial constitutive laws. Based on Roe linearization, this numerical method is well-suited to the present system of partial differential equations, and has been validated with the Riemann problem of nonlinear elastodynamics. Nonsmooth solutions such as shock waves are well-captured, even in the case of nonconvex stress-strain relationships. The method is adapted to a case with an additional scalar evolution equation, which is deduced from the internal-variable model [13] of slow dynamics. The numerical model reproduces qualitatively experimental observations related to dynamic acoustoelasticity.

We mention here a few improvements to be introduced. Boundary conditions such as free edges and oscillating walls need to be implemented so as to reproduce real configurations in a more realistic way (section 7 of [17]). Due to resonance, viscoelastic attenuation needs then to be incorporated in the model, which has been carried out simi- larly to [10] in recent works [32]. Currently, higher-order shock-capturing methods [33] are developed, as well as similar methods in multiple space dimensions.

\section{Appendix}

In the case of Murnaghan's law (12), strict hyperbolicity of (15) is ensured if

$\vartheta-2+6(1-\vartheta)(1+\varepsilon)^{2}+5 \vartheta(1+\varepsilon)^{4}>0$.

Therefore, one must have

$$
\varepsilon \in \begin{cases}] \varepsilon_{+}, \varepsilon_{-}[ & \text {if } \vartheta<0, \\ ] \varepsilon_{+},+\infty[ & \text { if } 0 \leqslant \vartheta<2, \\ ]-1, \varepsilon_{-}[\cup] \varepsilon_{+},+\infty[ & \text { if } 2 \leqslant \vartheta\end{cases}
$$

where

$$
\varepsilon_{ \pm}=-1+\sqrt{\frac{3(\vartheta-1) \pm \sqrt{9-8 \vartheta+4 \vartheta^{2}}}{5 \vartheta}}
$$

The constitutive law (12) is convex if $\sigma^{\prime \prime}(\varepsilon) \geqslant 0$, i.e.

$$
3+2 \vartheta+(3+12 \vartheta) \varepsilon+15 \vartheta \varepsilon^{2}+5 \vartheta \varepsilon^{3} \geqslant 0 .
$$

Hence, Murnaghan's law is locally concave at small strains ( $\varepsilon \simeq 0$ ) provided that $\vartheta \leqslant-3 / 2$, otherwise it is locally convex at small strains. The inflexion point

$$
\varepsilon_{0}=-1+\sqrt{\frac{3(\vartheta-1)}{5 \vartheta}} .
$$

is represented in Figure 4, as well as the hyperbolicity domains (A2) of Murnaghan's law.

\section{Acknowledgments}

This work was supported by the interdisciplinary mission of CNRS (INFINITI). The project leading to this publication has received funding from Excellence Initiative of Aix-Marseille University - A*MIDEX, a French "Investissements d'Avenir" programme. It has been carried out in the framework of the Labex MEC. Opération réalisée avec le concours du Programme d'investissements d'avenir du Gouvernement français dont la gestion a été confiée à l'Andra.

\section{References}

[1] R. A. Guyer, P. A. Johnson: Nonlinear mesoscopic elasticity: evidence for a new class of materials. Phys. Today $\mathbf{5 2}$ (1999) 30-36.

[2] J. A. TenCate: Slow dynamics of earth materials: an experimental overview. Pure Appl. Geophys. 168 (2011) 22112219.

[3] G. Renaud, P.-Y. Le Bas, P. A. Johnson: Revealing highly complex elastic nonlinear (anelastic) behavior of Earth materials applying a new probe: Dynamic acoustoelastic testing. J. Geophys. Res.-Sol. Ea. 117 (2012) B06202. 
[4] J. Rivière, G. Renaud, R. A. Guyer, P. A. Johnson: Pump and probe waves in dynamic acoustoelasticity: comprehensive description and comparison with nonlinear elastic theories. J. Appl. Phys. 114-5 (2013) 054905.

[5] K. E. A. Van Den Abeele: Elastic pulsed wave propagation in media with second- or higher-order nonlinearity. Part I. Theoretical framework. J. Acoust. Soc. Am. 99-6 (1996) 3334-3345.

[6] C. Payan, V. Garnier, J. Moysan, P. A. Johnson: Applying nonlinear resonant ultrasound spectroscopy to improving thermal damage assessment in concrete. J. Acoust. Soc. Am. 121-4 (2007) EL125-EL130.

[7] T. Meurer, J. Qu, L. J. Jacobs: Wave propagation in nonlinear and hysteretic media-a numerical study. Int. J. Solids Struct. 39-21 (2002) 5585-5614.

[8] Y. Li, O. Bou Matar, B. Li, X. Chen: Pseudo-spectral simulation of 1D nonlinear propagation in heterogeneous elastic media. Wave Motion 52 (2015) 54-65.

[9] O. O. Vakhnenko, V. O. Vakhnenko, T. J. Shankland, J. A. Ten Cate: Strain-induced kinetics of intergrain defects as the mechanism of slow dynamics in the nonlinear resonant response of humid sandstone bars. Phys. Rev. E 70 (2004) 015602.

[10] N. Favrie, B. Lombard, C. Payan: Fast and slow dynamics in a nonlinear elastic bar excited by longitudinal vibrations. Wave Motion 56 (2015) 221-238.

[11] V. Lyakhovsky, Y. Ben-Zion, A. Agnon: Distributed damage, faulting, and friction. J. Geophys. Res.-Sol. Ea. 102B12 (1997) 27635-27649.

[12] V. Lyakhovsky, Z. Reches, R. Weinberger, T. E. Scott: Nonlinear elastic behaviour of damaged rocks. Geophys. J. Int. 130-1 (1997) 157-166.

[13] H. Berjamin, N. Favrie, B. Lombard, G. Chiavassa: Nonlinear waves in solids with slow dynamics: an internalvariable model. Proc. R. Soc. A 473 (2017) 20170024.

[14] G. A. Maugin, W. Muschik: Thermodynamics with internal variables. Part I. General concepts. J. Non-Equilib. Thermodyn. 19 (1994) 217-249.

[15] G. A. Maugin: The saga of internal variables of state in continuum thermo-mechanics (1893-2013). Mech. Res. Commun. 69 (2015) 79-86.

[16] E. Godlewski, P.-A. Raviart. Numerical Approximation of Hyperbolic Systems of Conservation Laws. Springer, 1996.

[17] R. J. LeVeque. Finite-Volume Methods for Hyperbolic Problems. Cambridge University Press, 2002.
[18] R. Velasco-Segura, P. L. Rendón: A finite volume approach for the simulation of nonlinear dissipative acoustic wave propagation. Wave Motion 58 (2015) 180-195.

[19] F. D. Murnaghan: Finite deformations of an elastic solid. Am. J. Math. 59 (1937) 235-260.

[20] L. A. Ostrovsky, P. A. Johnson: Dynamic nonlinear elasticity in geomaterials. Riv. Nuovo Cimento 24-7 (2001) 1-46.

[21] C. Payan, V. Garnier, J. Moysan, P. A. Johnson: Determination of third order elastic constants in a complex solid applying coda wave interferometry. Appl. Phys. Lett. 94 (2009) 011904.

[22] H. Berjamin, B. Lombard, G. Chiavassa, N. Favrie: Analytical solution to 1D nonlinear elastodynamics with general constitutive laws. Wave Motion 74 (2017) 35-55.

[23] R. W. Ogden: Non-Linear Elastic Deformations. Ellis Harwood Ltd, 1984.

[24] G. A. Holzapfel: Nonlinear Solid Mechanics: A Continuum Approach for Engineering. John Wiley \& Sons, 2000.

[25] D. S. Drumheller: Introduction to wave propagation in nonlinear fluids and solids. Cambridge University Press, 1998.

[26] A. N. Norris: Finite-amplitude waves in solids. In: Nonlinear Acoustics (eds M. F. Hamilton, D. T. Blackstock), pp. 263-278. Academic Press, 1998.

[27] K. R. McCall: Theoretical study of nonlinear elastic wave propagation. J. Geophys. Res.-Sol. Ea. 99-B2 (1994) 25912600.

[28] P. A. Johnson, P. N. J. Rasolofosaon: Nonlinear elasticity and stress-induced anisotropy in rock. J. Geophys. Res.Sol. Ea. 101-B2 (1996) 3113-3124.

[29] A. Kurganov, G. Petrova, B. Popov: Adaptive semidiscrete central-upwind schemes for nonconvex hyperbolic conservation laws. SIAM J. Sci. Comput. 29-6 (2007) 2381-2401.

[30] A. Voss: Exact Riemann solution for the Euler equations with nonconvex and nonsmooth equation of state. $\mathrm{PhD}$ thesis. RWTH Aachen, 2005.

[31] H. Berjamin: RiemannElasto1D Toolbox (accessed 16 October 2017), https://fr.mathworks.com/matlabcentral/fileex change/63424/

[32] H. Berjamin, B. Lombard, G. Chiavassa, N. Favrie: Modeling longitudinal wave propagation in nonlinear viscoelastic solids with softening. Int. J. Solids Struct. (2018), in press.

[33] C.-W. Shu: High order weighted essentially nonoscillatory schemes for convection dominated problems. SIAM Rev. 51-1 (2009) 82-126. 